\title{
Numerical Analysis of Reynolds Equation for Gas Lubrication in a High 1 Region*
}

\author{
Nobuyoshi KAWABATA **
}

\begin{abstract}
In this paper, a new scheme is presented for solving the Reynolds equation for gas lubrication in a high $\Lambda$ region by the direct numerical solution method. This scheme derives from the concept that Poiseuille flow has an elliptical property and Couette flow has a parabolic one. This scheme has the following advantages: (1) High stability in a high $\Lambda$ region; (2) High precision in the case of coarse meshes; ( 3 ) Simple algebraical expression; (4) The required quantity of computer memory and cpu time is less than that required by other schemes; (5) There is no waving of pressure results, which is often seen in conventional finite element methods.
\end{abstract}

Key Words: Numerical Analysis, Lubrication Theory, Reynolds Equation, Gas Bearing, Direct Numerical Method, High Compressibility

\section{Introduction}

Gas bearings have superior qualities and are applied in various fields. The governing equation of the gas bearing film pressure is a nonlinear Reynolds equation. The pressure distribution of the gas lubricated fluid film has been analyzed by using a linearized approximation of the Reynolds equation. Recently, however, because of the development of computer performance, it is said that the direct numerical calculation of Reynolds equation is the most economical $^{(1)}$. But, in a high $A$ region at high speed, for a very small film thickness and a rarefied atmosphere, the nonlinearity of the Reynolds equation is very strong; so, the calculation of the Reynolds equation is largescale in such high $A$ regions.

The purpose of this study is to develop a new scheme of discretization, by which the Reynolds equation can be directly solved numerically and economically in a high 1 region. This scheme derives from the

* Receired 21 th February, 1986 Paper No.86-0146 A

* Faculty of Engineering, fukui University. :3 91 Bunkyo. Pukui, 910, Japan concept that the Poiseuille flow term of the Reynolds equation has an elliptical property, and a Couette flow by relative slide motion has a parabolic one. In this paper, it is shown that the film pressure distribution in a high $\Lambda$ region can be easily calculated by discretization using the present scheme. It is assumed that the film flow is laminar, the effect of the inertial force is negligible and the temperature of the gas film is constant.

\section{Nomenclature}

$a:$ wall collection factor.

$B$ : width of slider bearings.

$c:$ standard dimension of film thickness.

$h$ : film thickness.

II: normalized film thickness $=h / \mathrm{c}$.

$I$ : : length of slider bearing.

$M$ : Knudsen number of the ambient gas. (the suffixed value means a number of division).

$N$ : number of grooves of spiral grooved bearing.

$p$ : film pressure.

$f_{12}:$ ambient pressure.

$l^{\prime}:$ normalized pressure $=-p / p_{a}$.

$P_{i}:$ nomalized chamber pressure of circular spi- 
ral grooved bearing.

$P_{S}$ : normalized pressure at a step of slider bearing.

$Q:$ normalized mass flux $=q\left(12 \mu L / \rho_{a} c^{3} p_{a}\right)$.

$r_{i}, r_{0}$ : inner and outer radius of circular spiral grooved bearing.

$U:$ relative slide velocity of slider bearing.

$W:$ normalized load carrying capacity of circular spiral grooved bearlng $=w / \pi r_{o}^{2} p_{a}$.

$\beta$ : groove angle of circular spiral grooved bearing.

$\delta:$ groove depth of circular spiral grooved bearing.

$\Lambda$ : compressibility number $=6 \mu U L / p_{a} c^{2}$ (slider bearing) $=6 \mu \omega r_{o}^{2} / p_{a} c^{2} \quad$ (circular spiral grooved bearing)

$\omega$ : angular velocity of circular spiral grooved bearing.

\section{Discretization of Reynolds Equation}

\subsection{One-dimensional case}

An infinitely long and steady slider bearing of arbitrary film thickness is considered. The lubricated domain is divided at regular intervals and a part, as shown in Fig.l, is considered. The $X$-axis is taken in the direction of slide motion. The normalized mass flux $Q_{X}$ is expressed as follows.

$$
Q_{x}=-H^{3} P \frac{\partial P}{\partial x}+\Lambda H P
$$

The first term on the right hand side of Eq. (1) is a component of Poiseuille flow induced by a pressure gradient. The second term is a component of Couette flow induced by the relative slide motion between two bearing surfaces. If a linear pressure distribution is assumed in the interval between $W$ and $P$, the mass flux $Q_{x w}$ at section $w$ is expressed as follows.

$$
Q_{X w}=\left(\frac{H_{w}^{3} P_{w}}{\Delta X}+\frac{\Lambda}{2} H_{w}\right) P_{W}-\left(\frac{H_{w}^{3} P_{w}}{\Delta X}-\frac{\Lambda}{2} H_{w}\right) P_{P}
$$

On discretizating $Q_{x}$ e similarly, the continuity equation is given, as follows, by equalizing $Q_{x w}$ to $Q_{x} e$.

$$
\left\{\frac{H_{w}^{3} P_{w}}{\Delta X}+\frac{H_{e}^{3} P_{e}}{\Delta X}-\frac{\Lambda}{2}\left(H_{w}-H_{e}\right)\right\} P_{P}
$$

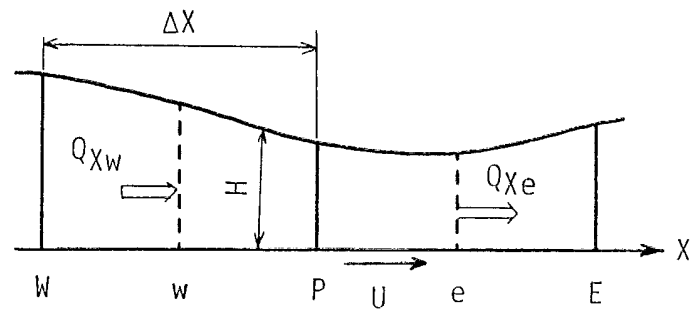

Fig. 1 One-dimensional film thickness.

$$
=\left(\frac{H_{w}^{3} P_{w}}{\Delta X}+\frac{\Lambda}{2} H_{w}\right) P_{W}+\left(\frac{H_{e}^{3} P_{e}}{\Delta X}-\frac{\Lambda}{2} H_{e}\right) P_{E}
$$

The coefficient of $P_{E}$ in the second term on the right hand side of Eq. ( 3 ) becomes a negative value in a high $\Lambda$ region. In such cases, an increase in $P_{E}$ reduces $P_{P}$. As a result, an unnatural waving of pressure distribution appears. Therefore, in the case of solving the gas lubricated film pressure by Eq. (3), the range of mesh size $\Delta X$ must be selected as follows.

$$
\Delta X<\frac{2}{\Lambda} H^{2} P
$$

This means that the applicable range of $\Lambda$ is limited and the domain must be divided finely in reverse proportion to the $\Lambda$ value. Hence, calculation by Eq. (3) is not advantageous from the viewpoint of cpu time and wide use.

As the Poiseuille flow in the first term on the right hand side of Eq. (1) is induced by the pressure gradient, the pressure at the node is influenced equally by pressures at the neighboring nodes. Nevertheless, as the Couette flow in the second term is induced by a relative slide motion between two bearing elements, the pressure at the node is influenced more strongly by nodes on one side than on the other side. It is actually known that the effect of upstream side pressure becomes dominant in a high $\Lambda$ region. This is understandable since the Poiseuille term of the Reynolds equation has an elliptical property and the Couette term has a parabolic one. Accordingly, by

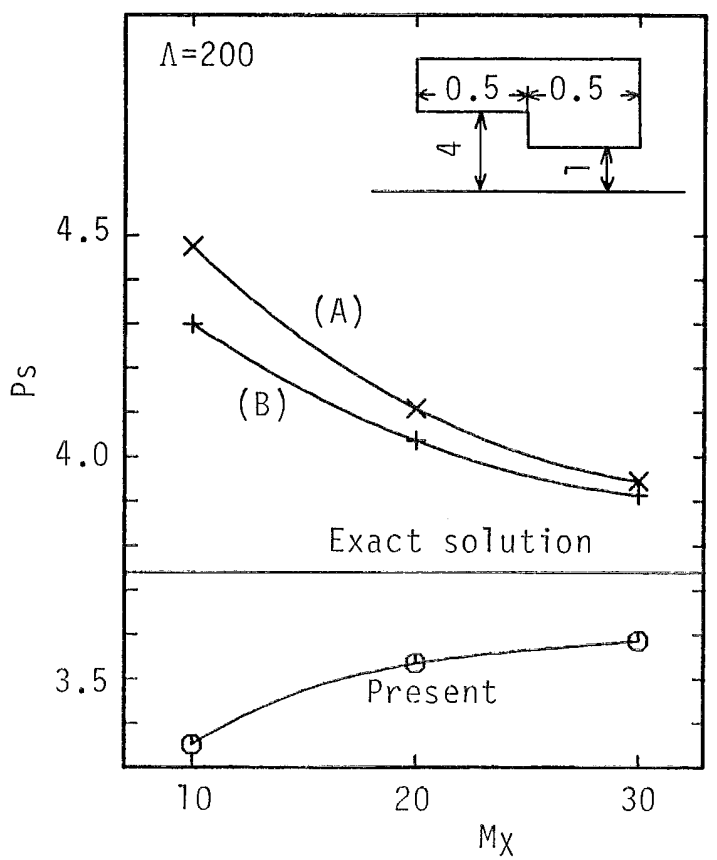

Fig. 2 Effect of the number of divisions $M_{s}$ on the step pressure $P_{s}^{\prime}$ in an infinitely long-stepped slider bearing. 
substituting the pressure $P_{W}$ at the upstream side node for the pressure $P$ in the second term on the right hand side of Eq. (1), Qxe is expressed as follows.

$$
Q_{X w}=\left(\frac{H_{w}^{3} P_{w}}{\Delta X}+\Lambda H_{w}\right) P_{w}-\frac{H_{w}^{3} P_{w}}{\Delta X} P_{P}
$$

$Q_{x e}$ is discretized similarly, and by substituting $Q_{x w}$ and $Q_{x e}$ into the continuity equation $Q_{x w}=Q_{x e}$, the algebraic equation for node pressures is obtained as

$$
\begin{aligned}
& \left(\frac{H_{w}^{3} P_{w}}{\Delta X}+\frac{H_{e}^{3} P_{e}}{\Delta X}+\Lambda H_{e}\right) P_{P} \\
& \quad=\left(\frac{H_{w}^{3} P_{w}}{\Delta X}+\Lambda H_{w}\right) P_{W}+\frac{H_{e}^{3} P_{e}}{\Delta X} P_{E}
\end{aligned}
$$

Coefficients of $P_{W}, P_{P}$, and $P_{E}$ are positive in a high $\Lambda$ region, so the unnatural waving of pressure distribution does not appear.

The following two methods are quoted in reference (2) as methods of discretization for a high $\Lambda$ region: (A) a nonlinear approximation of a pressure gradient by analytical solution (Harrison's equation) and a successive stepped film thickness; and (B) Elrod's nonlinear approximation (Exponential Approximation) by use of mean film thickness. Comparisons of methods (A) and (B) with the present scheme, and the relations between the pressure at step $P_{S}$ and the number of divisions $M_{X}$ for the infinitely long-stepped slider bearing are shown in Fig.2. It is clear in Fig.2 that the results obtained by the present scheme are nearer to the exact solution than the results obtained by (A) or (B). Methods (A) and (B) give a higher pressure while the present scheme

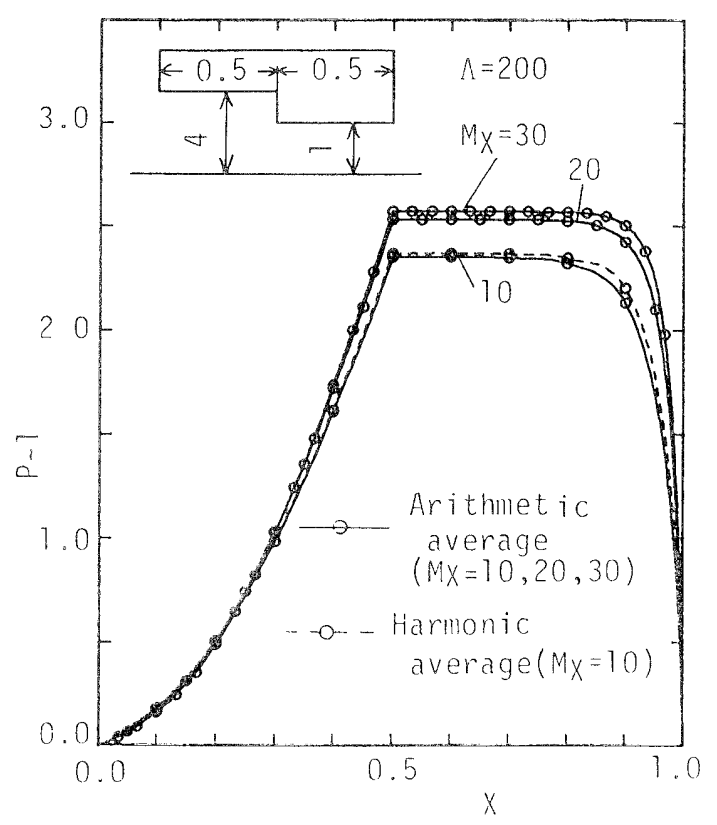

Fig. 3 Pressure distributions of an infinitely long-stepped slider bearing by using $l^{\prime}$ and $l^{\prime}$, by an arithmetic average and a hamonic average of node pres. sures. gives a lower pressure, than exact solution.

The results in Fig.2 are obtained by using $P_{w}$ and $P_{e}$ given by an arithmetic average of node pressures on both sides. However, if it is considered that $P_{H}$ becomes constant in the case of $\Lambda \rightarrow \infty$, the use of the harmonic average may be more suitable than the arithmetic average. Differences in pressure distributions by the arithmetic average and the harmonic average are shown in Fig.3. The dashed line means the result obtained by harmonic average $\left(M_{X}=10\right)$, and the solid line means the result obtained by arithmetic average ( $\left.M_{X}=10,20,30\right)$. Differences hardly exist in the domain before the step. In the domain near the out flow end, the result obtained by harmonic average is higher and more closely approaches the result obtained by fine mesh than the result obtained by arithmetic average.

\subsection{Two-dimentional case}

The present scheme can be easily extended to two- dimensional problems. If an arbitrary node (point $P$ ) and four neighboring nodes $(W, E, S, N)$ are considered in a mesh with regular intervals, fluxes which flow in and out of the element are calculated, as shown by the dashed lines in Fig.4, where, $w, e, s$ and $n$ are the cross points of boundaries of the element and grid lines.

Similar to Eq. (5), Qxw, which flows in the element through the $w$-boundary, is expressed as follows.

$$
\left.\begin{array}{l}
\Lambda_{x w}>0 \\
Q_{X w}=\Delta Y\left\{\left(\frac{H_{w}^{3} P_{w}}{\Delta X}+\Lambda_{x w} H_{w}\right) P_{w}-\frac{H_{w}^{3} P_{w}}{\Delta X} P_{P}\right\} \\
\Lambda_{X w}<0 \\
Q_{X w}=\Delta Y\left\{\frac{H_{w}^{3} P_{w}}{\Delta X} P_{w}-\left(\frac{H_{w}^{3} P_{w}}{\Delta X}-\Lambda_{X w} H_{w}\right) P_{P}\right\}
\end{array}\right\}
$$

where $\Lambda_{x w}$ means $\Lambda$ at the $\mathrm{w}$-point, which is obtained by $X$-directional slider velocity. Although the sign of

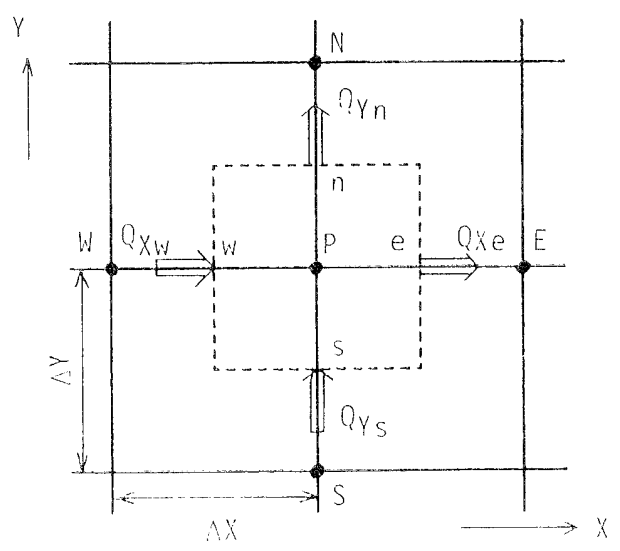

Fig. $t$ Two-dimensional mesh. 
$\Lambda_{x w}$ is usually known in fluid lubrication problems, in the case where the sign of $\Lambda_{X w}$ changes from node to node, Eq. ( 7 ) is also expressed as follows.

$$
\begin{aligned}
& Q_{x w}=\Delta Y\left\{\frac{H_{w}^{3} P_{w}}{\Delta X} \frac{H_{w}}{2}\left(\Lambda_{X w}+\left|\Lambda_{X w}\right|\right\} P_{W}\right. \\
& +\Delta Y\left\{-\frac{H_{w}^{3} P_{w}}{\Delta Y}+\frac{H_{w}}{2}\left(\Lambda_{X w}-\left|\Lambda_{X w}\right|\right)\right\} P_{P}
\end{aligned}
$$

Similarly, $Q_{X e}, Q_{Y S}$ and $Q_{Y n}$ are obtained. On substituting these fluxes into the continuity equation

$$
Q_{x w}-Q_{x e}+Q_{Y s}-Q_{Y n}=0
$$

and rearranging the pressures $P_{P}, P_{W}, P_{E}, P_{S}$ and $P_{N}$, based on the condition that $\Lambda_{X w}, \Lambda_{X e}, \Lambda_{Y s}$, and $\Lambda_{Y n}$ are positive, the algebraic equation for node pressure is obtained as follows.

$$
\begin{aligned}
a_{P} P_{P} & =a_{W} P_{W}+a_{E} P_{E}+a_{S} P_{S}+a_{N} P_{N} \\
a_{P} & =\Delta Y\left(\frac{H_{w}^{3} P_{w}}{\Delta X}+\frac{H_{e}^{3} P_{e}}{\Delta X}+\Lambda_{X e} H_{e}\right) \\
& +\Delta X\left(\frac{H_{s}^{3} P_{s}}{\Delta Y}+\frac{H_{n}^{3} P_{n}}{\Delta Y}+\Lambda_{Y n} H_{n}\right) \\
a_{W} & =\Delta Y\left(\frac{H_{w}^{3} P_{w}}{\Delta X}+\Lambda_{X w} H_{w}\right) \\
a_{E} & =\Delta Y \frac{H_{e}^{3} P_{e}}{\Delta X} \\
a_{S} & =\Delta X\left(\frac{H_{s}^{3} P_{s}}{\Delta Y}+\Lambda_{Y S} H_{s}\right) \\
a_{N} & =\Delta X \frac{H_{n}^{3} P_{n}}{\Delta Y}
\end{aligned}
$$

\section{Examples of Calculation}

\section{1 Slider gas bearing}

In this section, examples for slider gas bearings are shown and comparisons are made between the results obtained by the present scheme and those obtained by the finite element method. A taper flat slider gas bearing, whose dimensions are shown as Fig.5, is considered. Slider bearings are employed in magnetic recording disk systems, where the slider flying spacing is reduced to submicron values to realize high recording density. In such cases, the influence of the molecular mean free path appears in addition to that of a high $\Lambda$. So, a film pressure distribution is normally calculated using the modified Reynolds equation introduced by Burgdorfer ${ }^{(3)}$, which considers slip flow effects, and its propriety is clarified experimentally ${ }^{(4)(5)}$. As the slip flow term of the modified

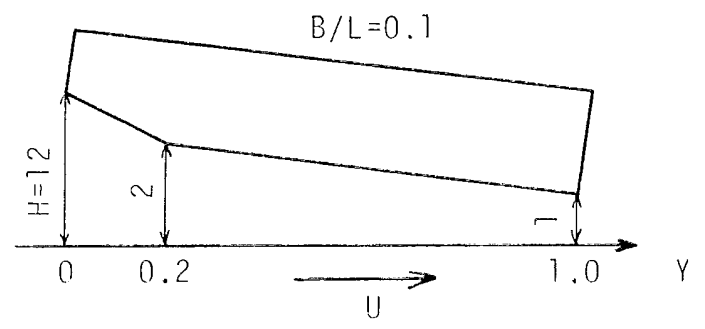

Fig. 5 Taper Hat slider bearing.
Reynolds equation has an elliptic property, it is discretized by means of a central difference. The film thickness is approximated by successive steps, and the discrete nonlinear algebraic equation is solved by the successive over-relaxation method.

In Fig.6, the effect of the number of divisions, $M_{Y}$, in the sliding direction is shown for the pressure distribution on the center-line of the slider in the cases of (a) $\Lambda=1000$ and (b ) $\Lambda=10000$. It is necessary that the taper part, in which the film thickness varies steeply is divided more finely than the flat part. For

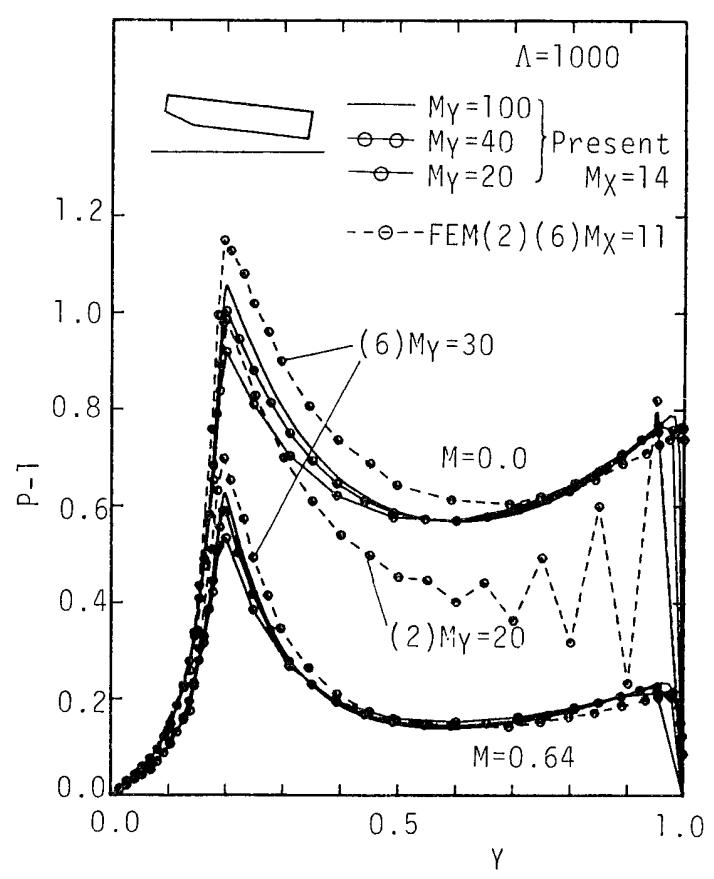

(a) $\Lambda=1000$

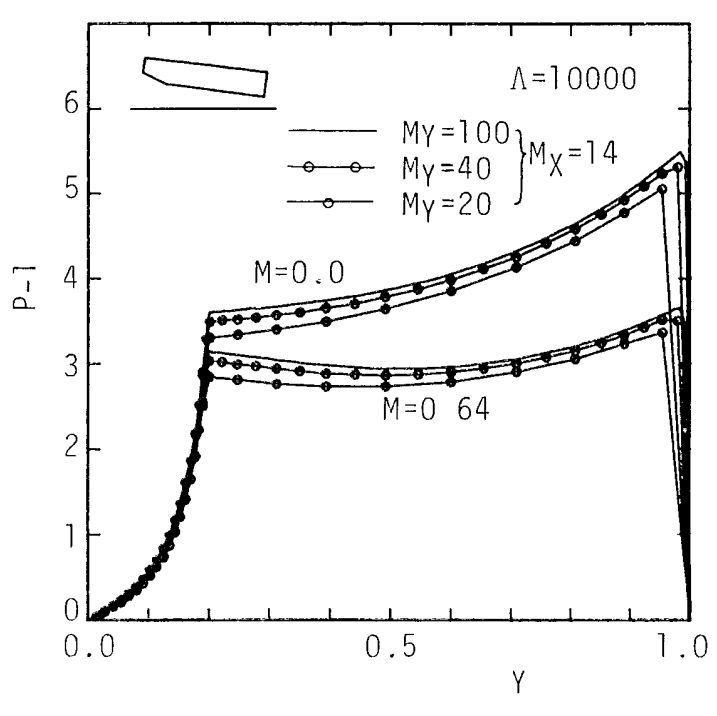

(b) $\quad A=10000$

Fig. 6 Pressure distribution on the center-line of taper flat slider bearing. 
this reason, the taper part and the flat part are divided into $M_{Y} / 2$ elements, respectively, more finely near the taper-flat boundary and the inflow and outflow ends. The minimum mesh length is about a third of the maximum one. The dashed lines show the results obtained by the finite element method, and a comparison is made between the cases of uniform meshes (11 $\times 20)^{(2)}$ and nonuniform meshes which are divided finely near the outflow end ${ }^{(6)}$. It is understandable that the unnatural waving of pressure distribution at outflow end appears in results obtained by the finite element method using coarse mesh. To prevent this waving, it is necesary to use finer meshes near the outflow end. Meanwhile, the results obtained by the present scheme are not influenced significantly by the number of divisions, especially at the outfiow end. So, it is clarified that the present scheme makes it possible to calculate with high accuracy those cases involving coarse meshes.

The number of iterations in the successive overrelaxation method is shown in Table 1 . The judgment of convergence is made under the condition

$$
\left|\delta P_{\max } / P_{\max }\right|<10^{-6}
$$

where $\delta P_{\max }$ is the maximum quantity of pressure

Table 1 Number of iterations $\left(M_{X}=14\right)$.

\begin{tabular}{|c|c|c|c|c|c|c|c|c|}
\hline 1 & $M N Y$ & 20 & 30 & 40 & 50 & 60 & 80 & 100 \\
\hline \multirow{3}{*}{1000} & 0.0 & 201 & 215 & 232 & 274 & 343 & 438 & 668 \\
\cline { 2 - 9 } & 0.64 & 388 & 396 & 406 & 418 & 433 & 511 & 626 \\
\hline \multirow{3}{*}{10000} & 0.0 & 267 & 259 & 253 & 258 & 262 & 272 & 289 \\
\cline { 2 - 8 } & 0.64 & 267 & 263 & 266 & 272 & 282 & 310 & 348 \\
\hline
\end{tabular}

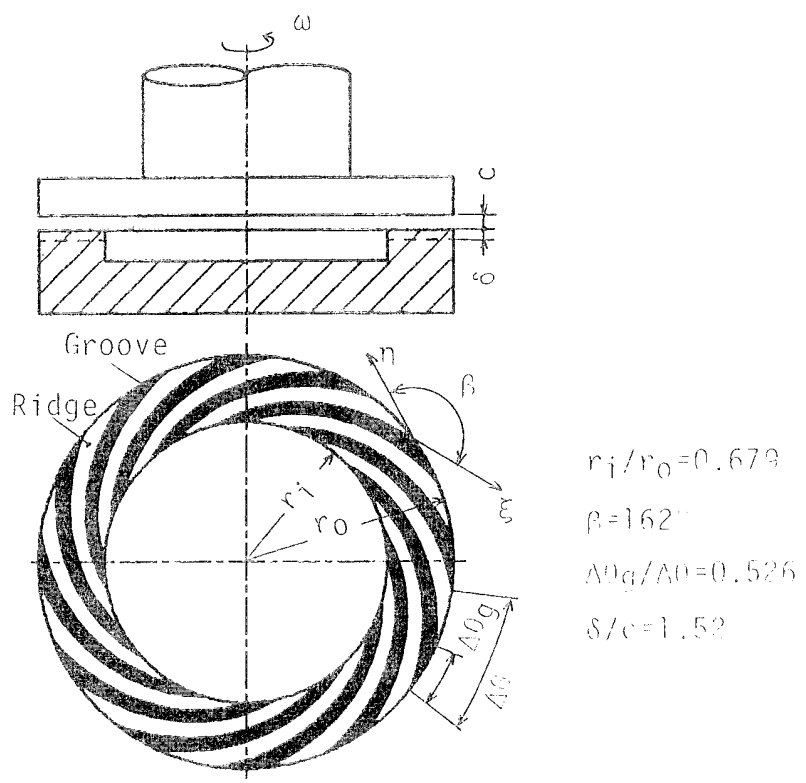

Mig. 7 Circular distip siral groved bearing. collection by an iteration and $P_{\max }$ is the maximum pressure. In Table 1 it is seen that the number of iterations increases in accordance with increases of the division number $M_{Y}$ in the sliding direction in the case of $\Lambda=1000$; but there are no significant increases in the case of $\Lambda=10000$. So, it is clarified that the cpu time obtained by the present scheme does not increase in a high $\Lambda$ region.

\section{2 Circular disk spiral grooved thrust bearings}

In this section, examples of calculations obtained by the present scheme are shown for a circular disk spiral grooved thrust bearing used in a rarefied atmosphere. A bearing whose dimensions are shown in Fig. 7 is considered. To deal easily with the configuration of the grooves, the fundamental equations are transformed to coordinates $(\xi, \eta)^{(7)}$, where $\xi$ is a coordinate along the groove-ridge boundary and $\eta$ is a coordinate along the sliding direction. The algebraic equation obtained by the present scheme is solved by the iter-

Table 2 Influences of $M_{\xi}, M_{\eta}$ on $W(\Lambda=200)$.

\begin{tabular}{|c|c|c|c|c|c|c|}
\hline$M_{\xi}$ & 10 & 16 & 20 & 30 & 40 & 50 \\
\hline$M_{n}$ & \multicolumn{6}{|c|}{20} \\
\hline$W$ & 3.055 & 3.160 & 3.183 & 3.219 & 3.236 & 3.246 \\
\hline$M_{\xi}$ & \multicolumn{4}{|c|}{30} & & \\
\hline$M_{n}$ & 10 & 14 & 20 & 30 & & \\
\hline$W$ & 3.278 & 3.246 & 3.219 & 3.226 & & \\
\hline
\end{tabular}

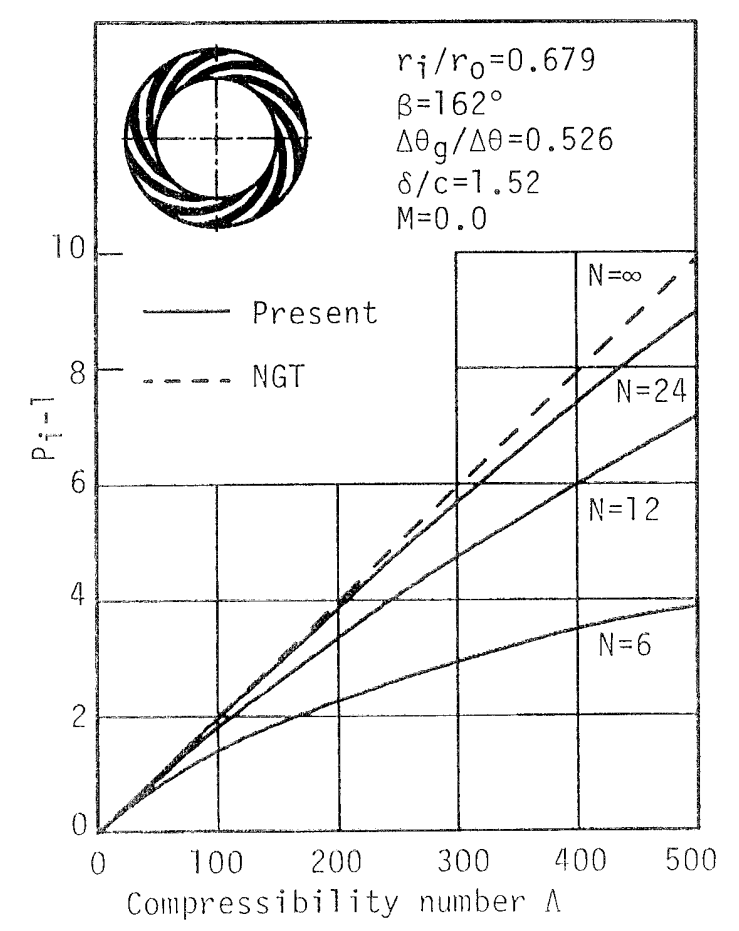

Fig. \& Elfect of the number of grooves $N$ on the chamber pressure $P^{\prime}$ in circular spiral groored bearing. 
ative successive over-relaxation method. In Table 2, the effect of the number of divisions $M_{\xi}$ and $M_{\eta}$ in the direction of $\xi$ and $\eta$, respectively, on the normalized

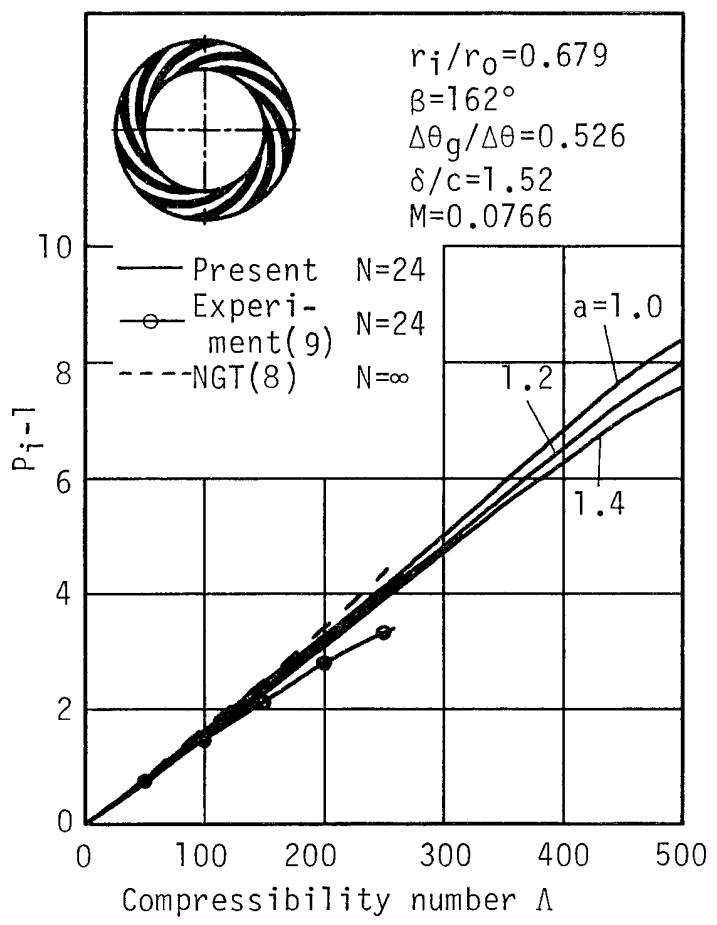

Fig. 9 Effect of $a$ on the chamber pressure $P_{i}$ in circular spiral grooved bearing.

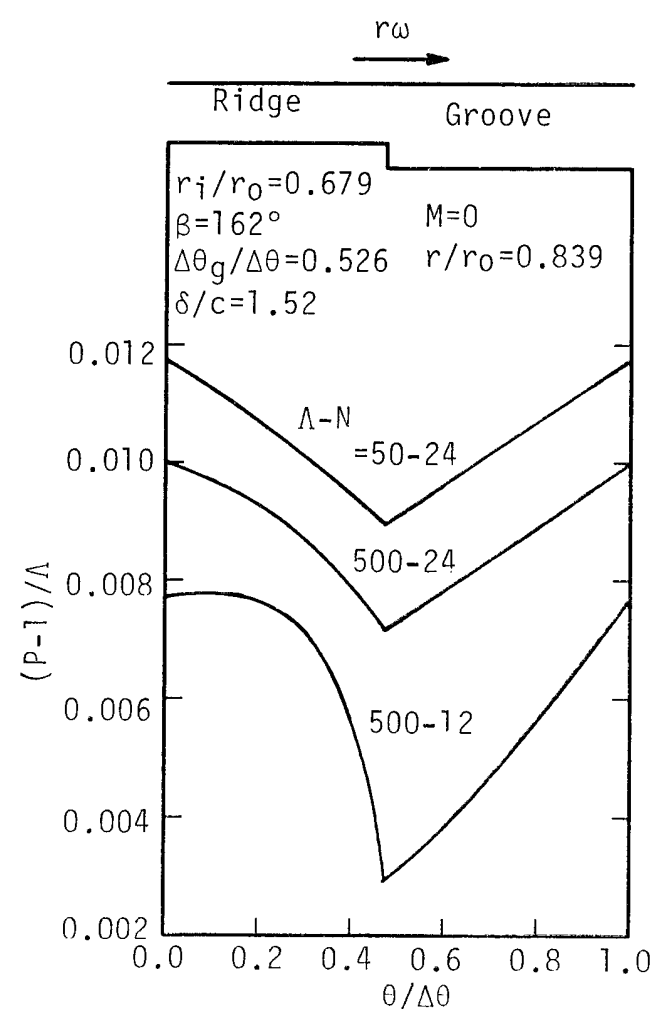

Fig. 10 Circumferential pressure distribution at the mean radius of circular spiral grooved bearing. load carrying capacity $W$ is shown for the case of $\Lambda$ $=200$. It is evident that there is a tendency for $W$ to approach an asymptic value, and the difference of $W$ between the coarsest mesh $(10 \times 20)$ and the finest mesh $(50 \times 20)$ is about $6 \%$. The following results are calculated by the use of nonuniform meshes $(30 \times 20)$ which are finer near the groove-ridge boundary and the outer and inner boundaries.

The effect of the number of grooves $N$ on the chamber pressure $P_{i}$ is shown in the case where the Knudsen number $M$ equals zero, as in Fig.8. The dashed line shows a result obtained by the approximate method using narrow groove theory (N.G.T. ${ }^{(8)}$, in which an infinite number of grooves is assumed. By this method, $P_{i}$ increases in proportion to $\Lambda$. Present results for a finite number of grooves give less chamber pressures than for the solution by N.G.T. This tendency becomes significant, according to an increase of $N$ or a decrease of $\Lambda$.

In Fig.9, comparisons between the present results and the experimental results obtained by Steranka ${ }^{(9)}$ are shown for the case of $M=0.0766$. The correction factor a depends on a working gas, and the quality and the mechanical character of the bearing surface, and has the effect of increasing the Knudsen number $M$ by $a$ times $^{(5)}$. Results from cases where $a=1,1.2$ and 1.4 are shown in Fig.9. Experimental results show a tendency for the rate of pressure increase to decrease, according to an increase of $\Lambda$; but the result obtained by N.G.T. ${ }^{(8)}$ shows the reverse tendency. Results from the Present scheme show a tendency similar to the experimental results, and it is clarified that this tendency increases according to increases in $a$, and that the present results approach the experimental results.

In Fig.10, the circumferential pressure distribution at the mean radius is shown. It is clarified that $\mathrm{N}$. G.T.'s assumption of a linear structure of pressure distribution on each groove or each ridge is realized, in the case of small $\Lambda$ and many $N$; but, with decreases in $N$ or increases in $\Lambda$, the assumption breaks, especially at the ridge.

\section{Conclusion}

In this paper, a new scheme is presented for solving the Reynolds equation for gas lubrication in a high $\Lambda$ region by the direct numerical solution method. This scheme has the following advantages:

(1) High stability in a very high $\Lambda$ region.

(2) High precision in the case of coarse meshes.

(3) Simple algebraical expression.

(4) The required quantity of computer memory and cpu time is less than that required by other schemes. 
Examples by the present scheme for a taper flat slider bearing and a circular disk spiral grooved thrust bearing are shown, and the following is clarified:

(1) There is no waving of pressure, which is often seen in conventional finite element solutions; and

(2) The effects of the groove number in a circular disk spiral grooved thrust bearing are clarified in the high $\Lambda$ region.

This research was supported financially, in part, by the Scientific Research-aid Fund (encouragement A60750134) by the Ministry of Education.

\section{References}

(1) Kaneko, R. and Mitsuya, Y., Jyunkatsu, (in Japanese), Vol. 20, No. 12 (1975), p. 883.

(2) Kaneko, R., Jyunkatsu, (in Japanese), Vol. 29, No.
9 (1984), p. 633

(3) Burgdorfer, A., Trans. ASME, Ser. D, Vo1. 81, No. 1 (1959), p. 94.

(4) Mitsuya, Y. and Kaneko, R., Bull. Jpn. Soc. Mech. Eng., Vol. 24, No. 187 (1981), p. 236.

(5) Ohkubo, T. and Mitsuya, Y., Trans. Jpn. Soc. Mech. Eng., (in Japanese), Vol.51, No.462, C (1985), p. 304

(6) Mitsuya, Y., Bull. Jpn, Soc. Mech. Eng., Vol. 22, No. 167 (1979), p. 863.

(7) Thompson, J. F., Thames, F. C. and Mastin, C. W., NASA CR-2729 (1977).

(8) Hsing, F. C. and Malanoski, S. B., Trans. ASME, Ser. F, Vol. 91, No. 1 (1969), p. 69.

(9) Steranka, P., M. T. I. Instrum. Laboratory Rept., E-2132 (1967). 